\title{
Letter
}

Andre-Pierre Blanchard-Dionne and Michel Meunier*

\section{Multiperiodic nanohole array for high precision sensing}

https://doi.org/10.1515/nanoph-2018-0108

Received July 26, 2018; revised October 10, 2018; accepted October 11,2018

\begin{abstract}
In this article, we present a multiperiodic nanohole array structure for improved sensing. The structure consists a series of rows of nanoholes, each having a different period in an ascending order. A monochromatic source illuminates the structure, and a resonance condition is met for the row having a momentum matching Bloch wave, which leads to extraordinary optical transmission. With this new plasmonic structure, the sensing signal can be retrieved using the spatial position of the transmission maxima. This setup requires a simple optical setup while achieving increased resolution and accuracy. A resolution of $4.6 \times 10^{-6}$ refractive index units is achieved, which is comparable to surface plasmon resonance system based on the Kretchmann configuration.
\end{abstract}

Keywords: nanomaterials; optical sensors; subwavelength structure; surface waves.

\section{Introduction}

Ever since nanohole arrays were shown to exhibit extraordinary optical transmission [1] and were used for sensing [2], there has been a considerable amount of research done to demonstrate their potential as biomedical diagnostic tools. This type of sensor is particularly well suited for highly multiplexed sensing, as the arrays can easily be integrated in an imaging system, taking advantage of linear collimation. This leads to the possibility of building a compact, low-cost, and fully integrated device that can achieve multiple detections using a lab-on-a-chip device [3]. But early demonstrations of sensing using those devices showed

*Corresponding author: Michel Meunier, Laser Processes and Plasmonics Laboratory, Engineering Physics Department, Polytechnique Montreal, 2900 Edouard Montpetit Blvd, Montreal H3T1J4, Canada, e-mail: michel.meunier@polymtl.ca Andre-Pierre Blanchard-Dionne: Laser Processes and Plasmonics Laboratory, Engineering Physics Department, Polytechnique Montreal, 2900 Edouard Montpetit Blvd, Montreal H3T1J4, Canada several challenges [4], particularly in terms of resolution, which ranged between $1 \times 10^{-4}$ and $1 \times 10^{-5}$ refractive index units (RIU) [5-9], compared to analogous devices based on surface plasmon resonance (SPR), which typically range at $1 \times 10^{-6}-1 \times 10^{-7}$ RIUs. A number of authors have implemented different strategies in order to improve the resolution of the device, such as crossed polarizers [10], Bragg mirrors on the edges of the holes [11], different shapes such as double-hole arrays [12] or elliptical holes [13], dual periodic system $[14,15]$, or dual wavelength system $[7,16]$, or used a different collimation angle [17]. Even with all these methods, most works on nanoholes arrays still rely on simplistic interrogation methods like a wavelength interrogation via a spectrometer $[9,18-20]$ or direct intensity measurements [21-24].

If we look at typical SPR sensors based on the Kretchmann configuration, usually three types of interrogation [25] are used. The first one, an intensity scan at a fixed angle, measures the difference in intensity of the reflected monochromatic light after a change in refractive index. It is done at a specific angle, which leads to SPR. A wavelength scan consists of measurements of the reflection of polychromatic light at a specific angle and uses the position of a maxima or minima of the spectrum for sensing. The angular scan uses the measurements of reflected light of a single wavelength at different angles around the angle of resonance and also uses a minima as the sensing signal. As was pointed out by a theoretical analysis of SPR sensor's performances [26], these two last interrogation methods usually lead to better performances, as the signal is not affected by correlated type noise. The angular scan is usually the method of choice, as it allows for a simpler optical setup. For nanohole-array-typed sensors, the picture is quite different, as the resonance is usually achieved using a linear collimation optical system. Angular scans are never used, and the systems rely on an intensity or wavelength interrogation.

\section{Approach and experimental setup}

In this paper, we present a fourth type of interrogation method, which consists in a scan of the hole array 
A

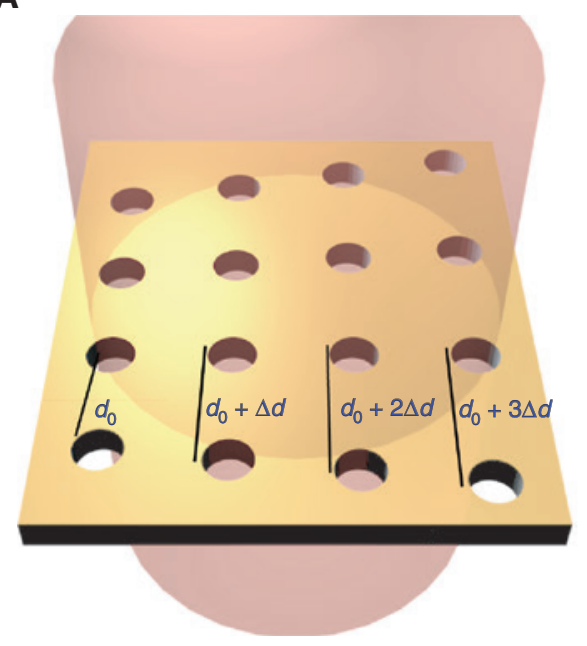

B
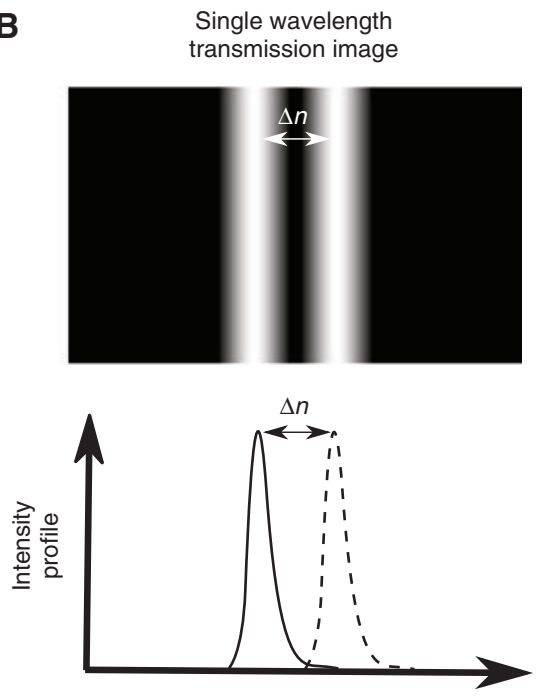

Figure 1: Schematic of the nanohole array structure and working principle.

(A) Multiperiodic nanohole array. Each column has a periodicity equal to $d_{0}+m \Delta d$, where $d_{0}$ is the initial period, $m$ is the number of the column, and $\Delta d$ is the increment. (B) The transmission image of the structure at a single wavelength will exhibit a bright fringe for a column with periods that match the resonant condition of the array. Changing the refractive index will move the fringe, and the position of the maxima gives the sensing signal.

periodicity, as illustrated in Figure 1. As the mechanism of the coupling of incident light to surface waves relies on the diffraction of light by the array, changing the period will lead to a change of the diffracted light's wavevector. Obtaining the spatial intensity profile of the transmitted light for a structure with incremental increases of the period results in a wavevector scan, just like an incremental change of the wavelength or of the incident angle would. The optical setup used for this sensing device remains a very simple monochromatic microscope with high acquisition frequency and retains the capacity for high multiplexing.

The structure consists of circular hole arrays with a period varying from 500 to $600 \mathrm{~nm}$ with an increment of $1 \mathrm{~nm}$ and diameter of $165 \mathrm{~nm}$, as shown in Figure 2. The dimensions of the holes were optimized using the analytical formalism of coupled mode theory $[27,28]$ in order to improve the quality factor of the resonance peak. The increment as well as the spacing of the hole in the y direction, thus the ascending periodicity, was chosen in order to retrieve a satisfactory resonance profile on the camera at the chosen magnification, which allows multiple sensors to be monitored at the same time. The scanning electron microscope image shows the hole array, with the spacing in the y-axis fixed at $700 \mathrm{~nm}$. An optical microscope image in the inset of that figure shows the spatial dependency of the resonant conditions of the array, which follows the period of each line. The sample thus acts as its own monochromator, with the wavelength of transmission/reflection being associated with

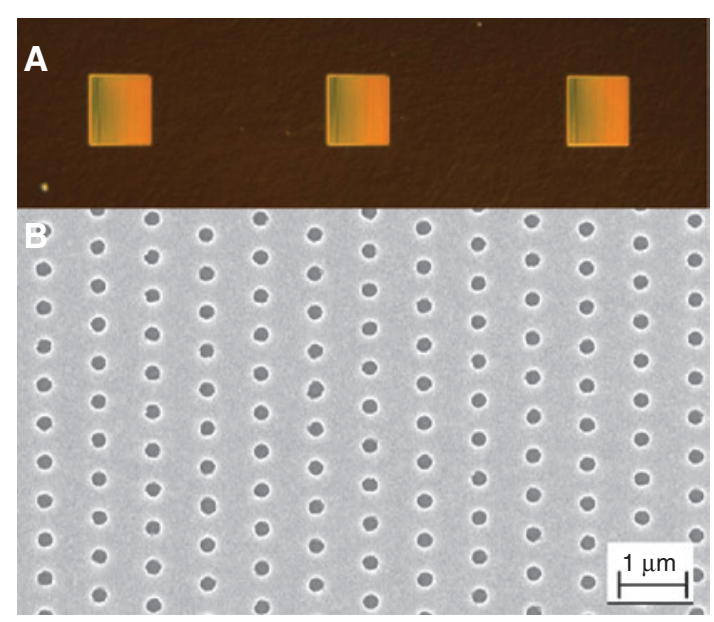

Figure 2: Images of the array.

(A) Reflection image of the multiperiodic nanohole array under white light illumination, using crossed polarizers. The sample reflects a different color, which is dependent of the periodicity. (B) SEM image of the nanohole array with a 1-nm increment changing period (the total sample goes from 500 to $600 \mathrm{~nm}$ ) in the x-axis and a 700-nm period in the $y$-axis. The hole size is $180 \mathrm{~nm}$, and the thickness of the gold film is $100 \mathrm{~nm}$.

a single line. The hole array was fabricated using E-beam lithography (Raith GmbH E-Line Plus, Dortmund, North Rhine-Westphalia, Germany) in a lift-off process [29]. The gold layer of $100 \mathrm{~nm}$ was deposited using an e-beam evaporator with a 2-nm Chrome adhesion layer. A polydimethylsiloxane microfluidic chip with three channels was used to deliver the solutions to the $3 \times 3$ sensor array. 

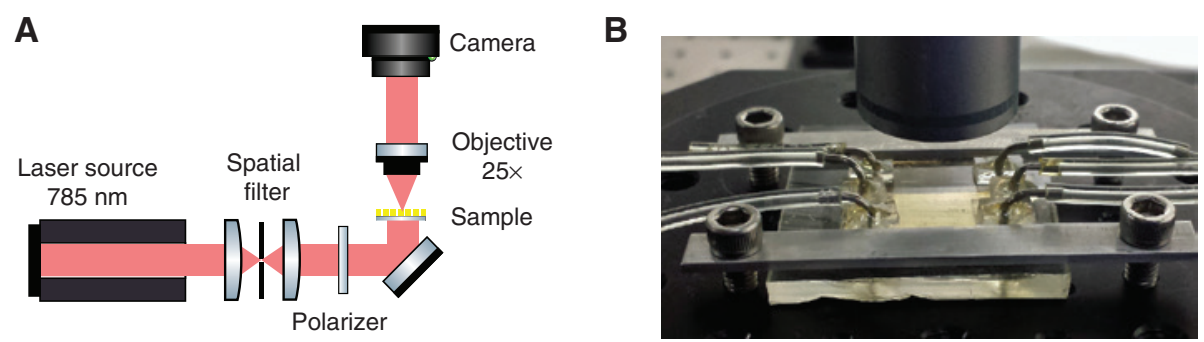

Figure 3: Optical setup and microfluidic chip.

(A) The optical setup consists of a homemade microscope with a monochromatic laser diode source, a spatial filter, and a polarizer. The microscope objective was $25 \times$. (B) The polydimethylsiloxane microfluidic chip with three parallel channels.

The sensor assembly was fixed to a rotary stage in order to align the samples with the beam.

The optical setup for sensing consists of a 785-nm laser diode source (Thorlabs Inc., Newton, NJ, USA) on a diode temperature controller (Newport Corporation, Irvine, CA, USA), which goes into a spatial filter (Newport Corporation, Irvine, CA, USA) towards the nanohole array sensors as illustrated in Figure 3. Light is collected via a microscope using a $15 \times$ microscope objective (Nikon) and a camera (Q-Imaging Corporate, Surray, British Columbia, Canada). The signal was analyzed in a Labview interface. A detection window was set around the transmission maxima of the sample, and the intensity was recorded as a function of the spatial position in the $\mathrm{x}$-axis, using a summation in the y-axis. The signal was recorded as a function of time, and the position of the maxima was determined using the centroid method. It should be noted that the resolution of the system depends on the magnification, which, in counter parts, limits the multiplexing capabilities of the device.

\section{Results and discussion}

In order to test the performances of the device, a sensitivity measurement as well as a real-time test of the limit of detection (LD) was performed. The sensitivity
A

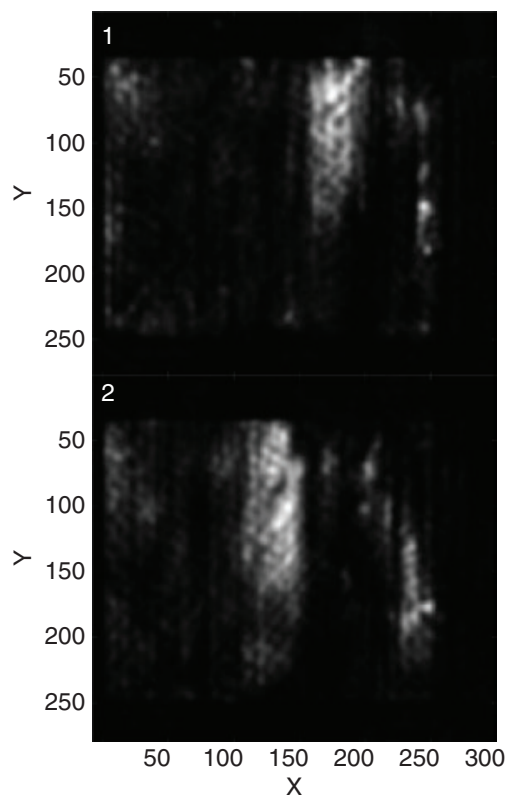

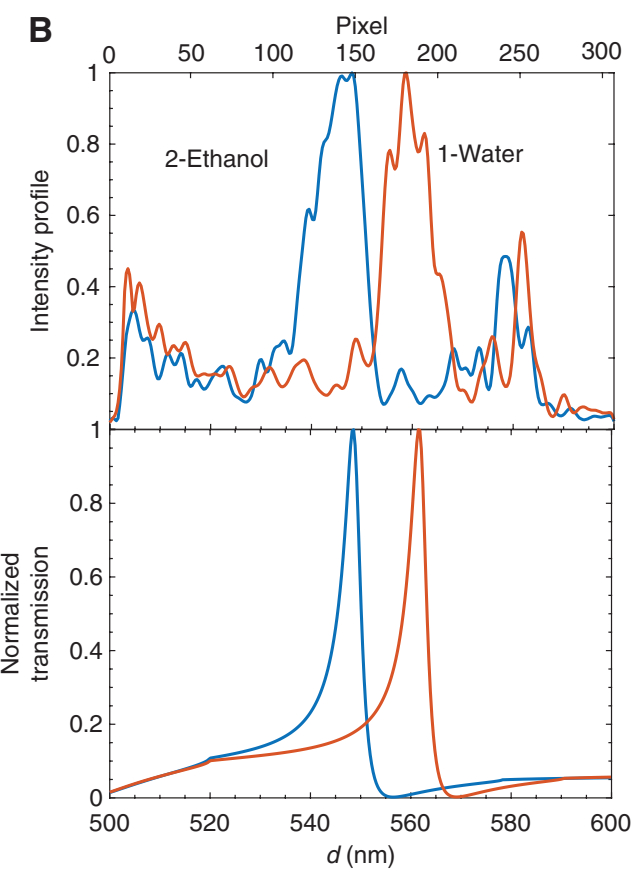

Figure 4: Sensitivity measurements.

Transmission image of the multiperiodic nanohole array sample with (1) water and (2) ethanol as the surrounding medium.

(B) Top Measured transmission profile of the images of the arrays. The intensity was integrated along the $y$-axis and plotted as a function of the $x$-axis. Bottom: Transmission intensity of the nanohole array as a function of the period as calculated using coupled mode theory. 


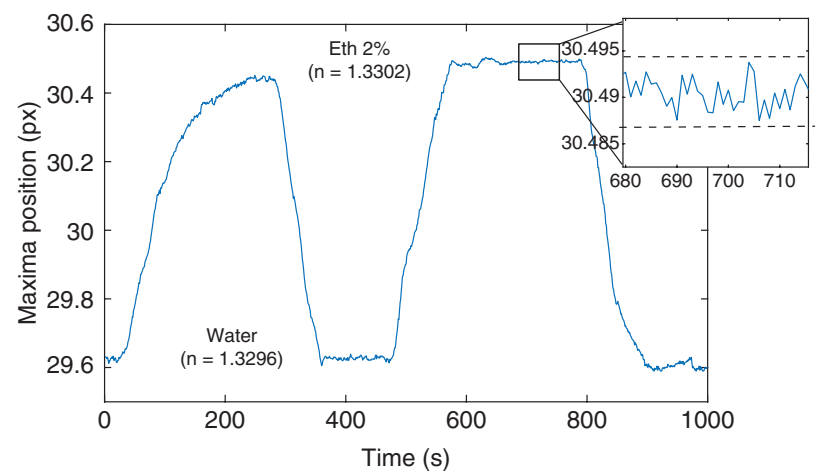

Figure 5: Limit of detection measurements. Real-time measurements of the position of the resonance maxima for different concentration of ethanol in water.

was measured by taking two images of the transmission image of the multiperiodic array with solutions of different refractive index, namely, water (1.3296 [30]) and ethanol (1.3577 [31]). The difference in the position of the maxima was determined to be at 38 pixels on the camera, as shown in Figure 4A. As the extent of the $70-\mu \mathrm{m}$ sample on the camera was at 300 pixels and that every line had a distance of $0.7 \mu \mathrm{m}$ and represented a shift of $1 \mathrm{~nm}$ of periodicity from one another, we can conclude that the sample's sensitivity was $S=1352 \mathrm{px} / \mathrm{RIU}=450.7 \mathrm{~nm} /$ RIU. Coupled mode theory $[27,28]$ was used in order to model the optical properties of the arrays as a function of the array period and to optimize the dimensions of the nanohole array. The transmission spectrum is given in Figure 4B, with a theoretical sensitivity of $462.6 \mathrm{~nm} / \mathrm{RIU}$, which is very similar to the one measured.

The LD of the system was measured by alternating solution from water to a small concentration of ethanol (2\%). The position of the maxima was recorded as a function of time using the centroid method. The sensing signal was temporally averaged to obtain one point every second. The LD of the systems corresponds to the smallest measurable minimal increment of refractive index that was detectable by the system, which we represent using $\mathrm{LD}=\frac{\Delta n}{\mathrm{SNR}}$, where $\Delta n$ is the refractive index change used in the experiment and SNR is the signal-to-noise ratio recorded. The noise was obtained using the standard deviation of the signal on a flat portion of the signal. For a change of refractive index of $5.6 \times 10^{-4} \mathrm{RIU}$, the SNR recorded was 122.7 for an LD of $4.6 \times 10^{-6}$ RIU. This is consistent with the LD obtained using the sensitivity and the noise level $(\sigma)$ of the system $(\mathrm{LD}=\sigma / S)$, which, if we look at the inset in Figure 5, can be evaluated to about $0.01 \mathrm{px}$.

\section{Conclusion}

The use of a multiperiodic nanohole array accounts for several advantages compared to multiplexed devices using a simple intensity measurement. First, as the signal relies on the position of a maxima, the monitoring of a change in refractive index will be directly accountable for the change in the resonant property of the system. This is important when we consider that the signal is obtained via transmission, which is susceptible to spurious noise by absorption in the sensing medium itself and by any contaminants in the solution or air bubbles. The resulting sensor is improved not only in its performances by reducing spurious noise and correlated noise (such as source noise) but also in accuracy. It also improves the multiplexing capabilities compared to systems using a wavelength interrogation measurement, as these systems either slow down greatly the acquisition rate by dividing it by the number of sensors or can only use one dimensional images, as the other dimension is used for the grating of the spectrometer. We thus believe that this new structure might become reliable in order to accomplish highresolution multiplexed sensors.

\section{References}

[1] Ebbesen TW, Lezec HJ, Ghaemi H, Thio T, Wolff P. Extraordinary optical transmission through sub-wavelength hole arrays. Nature 1998;391:667-9.

[2] Brolo AG, Gordon R, Leathem B, Kavanagh KL. Surface plasmon sensor based on the enhanced light transmission through arrays of nanoholes in gold films. Langmuir 2004;20:4813-5.

[3] Lopez GA, Estevez MC, Soler M, Lechuga LM. Recent advances in nanoplasmonic biosensors: Applications and lab-on-a-chip integration. Nanophotonics 2017;6:123.

[4] Dahlin AB, Wittenberg NJ, Höök F, Oh SH. Promises and challenges of nanoplasmonic devices for refractometric biosensing. Nanophotonics 2013;2:83-101.

[5] Hwang GM, Pang L, Mullen EH, Fainman Y. Plasmonic sensing of biological analytes through nanoholes. IEEE Sens J 2008;8:2074-9.

[6] Im H, Lee SH, Wittenberg NJ, et al. Template-stripped smooth ag nanohole arrays with silica shells for surface plasmon resonance biosensing. ACS Nano 2011;5:6244-53.

[7] Escobedo C, Vincent S, Choudhury A, et al. Integrated nanohole array surface plasmon resonance sensing device using a dual-wavelength source. J Micromechanics Microengineering 2011;21:115001.

[8] Lee KL, Chen PW, Wu SH, Huang JB, Yang SY, Wei PK. Enhancing surface plasmon detection using template-stripped gold nanoslit arrays on plastic films. ACS Nano 2012;6:2931-9. 
[9] Jia P, Jiang H, Sabarinathan J, Yang J. Plasmonic nanohole array sensors fabricated by template transfer with improved optical performance. Nanotechnology 2013;24:195501.

[10] Tetz KA, Pang L, Fainman Y. High-resolution surface plasmon resonance sensor based on linewidth-optimized nanohole array transmittance. Opt Lett 2006;31:1528-30.

[11] Lindquist NC, Lesuffleur A, Im H, Oh SH. Sub-micron resolution surface plasmon resonance imaging enabled by nanohole arrays with surrounding bragg mirrors for enhanced sensitivity and isolation. Lab Chip 2009;9:382-7.

[12] Lesuffleur A, Im H, Lindquist NC, Oh SH. Periodic nanohole arrays with shape-enhanced plasmon resonance as real-time biosensors. Appl Phys Lett 2007;90:243110.

[13] Tellez GAC, Tait RN, Berini P, Gordon R. Atomically flat symmetric elliptical nanohole arrays in a gold film for ultrasensitive refractive index sensing. Lab Chip 2013;13:2541-6.

[14] Blanchard-Dionne A, Guyot L, Patskovsky S, Gordon R, Meunier M. Intensity based surface plasmon resonance sensor using a nanohole rectangular array. Opt Express 2011;19:15041-6.

[15] Eitan M, Iluz Z, Yifat Y, Boag A, Hanein Y, Scheuer J. Degeneracy breaking of wood's anomaly for enhanced refractive index sensing. ACS Photonics 2015;2:615-21.

[16] Chang TY, Huang M, Yanik AA, et al. Large-scale plasmonic microarrays for label-free high-throughput screening. Lab Chip 2011;11:3596-602.

[17] de Menezes JW, Thesing A, Valsecchi C, Armas LE, Brolo AG. Improving the performance of gold nanohole array biosensors by controlling the optical collimation conditions. Appl Opt 2015;54:6502-7.

[18] Wittenberg NJ, Im H, Xu X, et al. High-affinity binding of remyelinating natural autoantibodies to myelin-mimicking lipid bilayers revealed by nanohole surface plasmon resonance. Anal Chem 2012;84:6031-9.

[19] Soler M, Belushkin A, Cavallini A, Kebbi-Beghdadi C, Greub G, Altug H. Multiplexed nanoplasmonic biosensor for one-step simultaneous detection of chlamydia trachomatis and neisseria gonorrhoeae in urine. Biosens Bioelectron 2017;94:560-7.

[20] Li X, Soler M, Özdemir Cl, Belushkin A, Yesilkoy F, Altug H. Plasmonic nanohole array biosensor for label-free and real-time analysis of live cell secretion. Lab Chip 2017;17:2208-17.

[21] Lesuffleur A, Im H, Lindquist NC, Lim KS, Oh SH. Laser-illuminated nanohole arrays for multiplex plasmonic microarray sensing. Opt Express 2008;16:219-24.

[22] Yang JC, Ji J, Hogle JM, Larson DN. Multiplexed plasmonic sensing based on small-dimension nanohole arrays and intensity interrogation. Biosens Bioelectron 2009;24:2334-8.

[23] Im H, Shao H, Park YI, Peterson VM, Castro CM, Weissleder R, Lee $\mathrm{H}$. Label-free detection and molecular profiling of exosomes with a nano-plasmonic sensor. Nat Biotechnol 2014;32:490.

[24] Im H, Lesuffleur A, Lindquist NC, Oh SH. Plasmonic nanoholes in a multichannel microarray format for parallel kinetic assays and differential sensing. Anal Chem 2009;81:2854-9.

[25] Piliarik M, Homola J. Spr sensor instrumentation. In: Homola J., ed. Surface Plasmon Resonance Based Sensors. New York, NY, USA, Springer, 2006, 95-116.

[26] Piliarik M, Homola J. Surface plasmon resonance (spr) sensors: approaching their limits? Opt Express 2009;17:16505-17.

[27] Blanchard-Dionne AP, Meunier M. Sensing with periodic nanohole arrays. Adv Opt Photonics 2017;9:891-940.

[28] Blanchard-Dionne AP, Meunier M. Optical transmission theory for metal-insulator-metal periodic nanostructures. Nanophotonics 2017;6:349-55.

[29] Blanchard-Dionne AP, Meunier M. Electron beam lithography using a pmma/p (mma $8.5 \mathrm{maa}$ ) bilayer for negative tone liftoff process. J Vacuum Sci Technol B Nanotechnol Microelectron Mater Process Meas Phenom 2015;33:061602.

[30] Hale GM, Querry MR. Optical constants of water in the 200-nm to 200- $\mu \mathrm{m}$ wavelength region. Appl Opt 1973;12:555-63.

[31] Rheims J, Köser J, Wriedt T. Refractive-index measurements in the near-ir using an abbe refractometer. Meas Sci Technol 1997;8:601. 